Mots. Les langages du politique

\title{
Mascarade de la diversité. Stéréotypes et feintises dans la série Inside Jamel Comedy Club
}

Nelly Quemener

\section{OpenEdition}

Journals

Édition électronique

URL : https://journals.openedition.org/mots/20698

DOI : $10.4000 /$ mots. 20698

ISSN : 1960-6001

Éditeur

ENS Éditions

Édition imprimée

Date de publication : 15 septembre 2012

Pagination : 113-126

ISBN : 9782847883732

ISSN : 0243-6450

Référence électronique

Nelly Quemener, « Mascarade de la diversité. Stéréotypes et feintises dans la série Inside Jamel Comedy Club », Mots. Les langages du politique [En ligne], 99 | 2012, mis en ligne le 15 septembre 2014, consulté le 22 avril 2022. URL : http://journals.openedition.org/mots/20698 ; DOI : https://doi.org/ $10.4000 /$ mots. 20698 


\section{Mascarade de la diversité. Stéréotypes et feintises dans la série Inside Jamel Comedy Club}

En juillet et août 2009, une série d'un genre nouveau est diffusée sur Canal +. Inside Jamel Comedy Club, classée parmi les docufictions, se compose de huit épisodes de 26 minutes et est produit par Kissmann Production, agence appartenant au comédien Jamel Debbouze. La série clôt un cycle de trois saisons du Jamel Comedy Club, émission de plateau diffusée pendant les étés 2006, 2007 et 2008, sur le même principe de huit épisodes par saison. Celle-ci consacre l'humour stand-up dont elle revendique la filiation anglo-saxonne et la rupture avec les codes du café-théâtre français ${ }^{1}$. Elle fait intervenir quatre jeunes humoristes par épisode, dans des sketches de cinq minutes joués sur le ton de la conversation avec le public. Portée par des comédiens de toutes origines sociales et ethnoraciales, elle ouvre un espace d'évocation des enjeux identitaires et des discriminations relatifs à la « race », à l'ethnicité, à la religion, au genre, au handicap ou à l'apparence physique. Plus qu'un commentaire d'actualité, elle fait de l'expérience vécue et du récit à la première personne l'origine du discours politique.

À l'instar de l'émission de plateau, la série Inside Jamel Comedy Club s'appuie sur la fabrique du vraisemblable et de l'authenticité. Elle suit le quotidien conflictuel d'une troupe de stand-up, composée en majorité des humoristes issus de la première saison de l'émission, parmi lesquels Thomas N'Gijol, Fabrice Éboué, Blanche, Yassine, Claudia, Amelle Chabi, Frédéric Chau, Wahid, Noom, Dédo. Elle s'apparente au making of d'une tournée à travers la France qui eut effectivement lieu en 2008 et conduisit à un DVD, Le Jamel Comedy Club au Casino de Paris. Malgré cette forme documentaire, la série satisfait les exigences

1. Issu des minorités raciales aux États-Unis et porté par des figures comme Lenny Bruce, Eddy Murphy et Will Smith, le stand-up consiste en des jeux sur les stéréotypes de « race », de genre et de classe à travers le récit d'expériences vécues et des saynètes du quotidien (Schulman, 1994, p. 108-109).

King's College de Londres

Laboratoire Communication, Information, Médias

nellyquemener@hotmail.com 
d'une tonalité humoristique en présentant des signes de fausseté et d'exagération. Elle bascule alors du côté de la représentation fictionnelle et resignifie les préjugés qui gouvernent les interactions dans une mise en scène hyperbolique des procédés de stéréotypisation. Inside Jamel Comedy Club se situe à la charnière des sitcoms autobiographiques héritières du stand-up² et des comédies filmées à la façon d'un documentaire, aussi appelées mocumentaires3.

Dans l'article qui suit, nous souhaitons revenir sur l'imbrication complexe des signes de la réalité et de la fiction dans Inside Jamel Comedy Club. Nous montrerons que le ressort humoristique s'appuie sur la combinaison d'une exagération des logiques d'exclusion et d'une authentification des personnages et des situations. Nous interrogerons ce dispositif humoristique à la manière d'une «micropolitique» (Foucault, 1994) par le truchement de laquelle, d’une part, les mécanismes de stéréotypisation sont mis à jour et, d'autre part, les moyens de la lutte contre ces mécanismes sont interrogés. La promotion de la diversité, fer de lance politique depuis 2006 en France dans le domaine des représentations audiovisuelles (Macé, 2007; Cervulle, 2011), est ici abordée de façon ironique et devient le lieu d'une confrontation quotidienne des différences individuelles et d'une fragmentation des enjeux collectifs (Gunster, 2005, p. 219). Une étude des commentaires formulés sur le site de partage de vidéos YouTube permettra d'identifier des modes de réception marqués par le rejet ou l'appropriation cathartique des stéréotypes, articulés autour de la croyance en la réalité/fiction de la série.

\section{L'impression de réalité et l'exagération humoristique}

Dans sa forme la plus courante, le genre docufiction consiste en la reconstitution d'un événement passé par des acteurs. Le classement parmi les docufictions d'Inside Jamel Comedy Club joue à la manière d'un trompe-l'œil. En effet, de prime abord, la série se présente plutôt comme un making of sur les coulisses du Jamel Comedy Club. Filmée caméra à l'épaule à la lumière naturelle lors des trajet en bus, lors des nuits à l'hôtel, lors des répétitions ou sur scène, elle s'empare des techniques du documentaire pour faire croire au spectateur qu'il assiste à des scènes spontanées et des rencontres fortuites habituellement inaccessibles 4 . Les situations du quotidien sont ponctuées de regards embar-

2. Larry David, corédacteur de la série Seinfeld, crée entre 2001 et 2010 une sitcom autobiographique, Curb your Enthousiam, filmée à la façon d'une téléréalité, dans laquelle il joue son propre rôle et se montre dans des situations cocasses du quotidien.

3. La plus célèbre est la série The Office produite par la BBC en 2004.

4. Nous qualifions le making of de documentaire en nous appuyant, d'une part, sur la classification floue de ces productions audiovisuelles par le CNC (Warigoda, 2010), d'autre part sur le fait que le making of constitue « une véritable mise en abyme de la fiction » (Hatchuel, 2009), dont la part documentaire repose sur «l'impression que des objets, entités, événements ou situations ont eu lieu ou ont existé dans le monde réel tel que représenté» (Gauthier, 2001 cité par Hatchuel). 
rassés lancés à la caméra ou de réactions parfois violentes et impulsives, émanant des personnes étrangères à la troupe ou des comédiens eux-mêmes. Ces adresses directes ou indirectes au cameraman et implicitement au spectateur ont pour fonction de rappeler qu'ils sont les témoins agissants d'une réalité en train de se faire. Les interviews des comédiens filmées face caméra qui viennent ponctuer les différents événements sont quant à elles inspirées de la téléréalité. L'ensemble du dispositif de la série constitue une "feintise énonciative» (Jost, 1995, p. 171). Il imite des genres télévisuels qui prétendent rendre compte de ce qui se passe «réellement » en coulisses et révéler l'intimité de personnes appartenant à notre monde (ibid.). La représentation crue et sans concession des membres de la troupe, apparemment filmés «sur le vif » et sous un jour peu gratifiant, est essentielle à la fabrication de cette impression de spontanéité. Amelle, jeune femme exigeante et sûre de ses charmes, s'énerve par exemple contre le maître d'hôtel à cause de son brushing raté. Thomas N'Gijol, obsédé sexuel, abuse de mineures et de femmes fragiles. Ces scènes activent un ressort du rire qui mêle grotesque bakhtinien et culte du banal (Bakhtine, 1970, p. 27-29; Jost, 2007) : elles dégradent l'image scénique des membres de la troupe en les présentant comme des individus «bassement» ordinaires.

La série semble s'adresser de façon privilégiée aux publics fans ou familiers de l'émission Le Jamel Comedy Club à même d'identifier ces renversements d'image et d'en apprécier la teneur humoristique (Sepulchre, 2007). Cette familiarité conditionne le repérage de certaines incohérences. Les personnages secondaires sont par exemple joués par des acteurs des saisons 2 et 3 qui, tout en gardant leurs noms de scène, adoptent des rôles fictifs. Jean-François, alias JF, le régisseur de la tournée dans la série, est interprété par Jean-François Cayrey, humoriste de la saison 2. Pour les spectateurs réguliers, ce type d'éléments peut servir d'indicateur de la facticité du programme. Il insiste néanmoins sur la forme plutôt que sur le fond et détourne l'attention de la dimension fictionnelle des événements racontés. Le scénario contribue en outre à lisser les divers rebondissements de telle sorte qu'ils s'enchaînent de façon apparemment naturelle. L'épisode 2 d'Inside Jamel Comedy Club commence par l'annonce du retrait de la tournée de Jamel Debbouze, médiateur et producteur du spectacle. Vécu comme un abandon, ce départ sert de révélateur et de déclencheur des intérêts individuels et des querelles d'ego. L'absence effective de Debbouze, fortement impliqué dans le projet, pendant la tournée peut apparaître difficilement plausible. Mais la série étouffe ce signe évident de fiction en le recouvrant du sceau du mystère. Elle active l'imaginaire de la star inaccessible et du patron impliqué dans des luttes d'intérêt supérieur. Elle transforme alors une situation improbable en situation vraisemblable et maintient le spectateur dans une incertitude permanente quant à la véracité des faits racontés.

La visée humoristique exige paradoxalement que la série signale sa fiction. Celle-ci se révèle dans les effets d'exagération et la caricature, particulièrement 
sensibles dans la succession des épisodes. Tous les personnages se voient attribuer un rôle caricatural au sein de cette comédie humaine et sociale. Yassine incarne l'enfant humble et naïf, Fabrice Éboué le chef autoritaire et injuste, Wahid le sous-fifre lèche-bottes, Claudia la mère protectrice, Frédéric Chau le bouc émissaire. La dynamique du groupe stabilise au fil des épisodes les traits de caractère des uns et des autres et met en exergue leur dimension fabriquée. Cantonnés dans leurs rôles respectifs, sans nuance même dans les moments les plus dramatiques tels que la mort de l'un d'entre eux, leurs réactions deviennent prévisibles et transforment les scènes du quotidien en mascarade révélatrice des logiques de pensée et de fonctionnement de chacun. Dès le premier épisode, Fabrice Éboué, de loin le personnage le plus manipulateur et narcissique de la troupe, évoque les salaires de chacun sur un ton de dénigrement :

Moi ce qui me choque, c'est pas qu'on soit mal payé. On bosse pour des Marocains, je m'attendais pas à mieux. Mais c'est qu'on soit tous payé pareil. Moi comme je l'ai dit, j'ai dix ans de café-théâtre [...]. J'estime que je dois pas être payé pareil que le Chinois qui y'a encore deux ans était steward.

Non seulement Éboué dévalorise sans sourciller ses camarades, mais il ponctue ses propos de stéréotypes racistes - les Marocains sont associés à la pauvreté et à l'avarice, tandis que Frédéric Chau est «le Chinois», désignant qui réduit l'acteur à sa catégorie ethnoraciale. Deux éléments accentuent le sentiment d'exagération. Le premier, lié au montage de l'émission, consiste en l'ajout d'un fond de musique douce et légère contrastant avec la violence des propos. Le second repose sur la dissonance entre ceux-ci et le personnage, homme métis, lui-même potentielle cible du racisme.

Dans cette scène comme dans beaucoup d'autres, les traces de fabrication et d'artifice reposent sur un jeu subtil du montage, du scénario et de la mise en scène et créent les possibilités d'une distanciation vis-à-vis des personnages et de leurs propos. L'épisode 1 nous apprend que Fabrice Éboué écrit les sketches de Claudia Tagbo, femme noire de la troupe. Dans l'une des scènes, il lui demande de "garder son côté tribal», afin de faire «voyager les spectateurs : l'Afrique, les bananes Plantin [sic], tout ça». La réaction interloquée de Claudia, filmée en plan rapproché, signale l'usage d'un imaginaire exotique. À l'échelle de la série, le montage, la caméra arrêtée sur les visages et le scénario exacerbent une situation d'humiliation de telle sorte qu'ils découvrent les logiques de pouvoir à l'œuvre. Un autre exemple est celui de Jamel Debbouze, dépeint comme un producteur sans scrupule qui exploite ses salariés. Lorsque les membres de la troupe décident de façon solidaire de s'élever contre le faible montant de leurs cachets, Debbouze, entouré et choyé par la production, s'énerve, use du chantage affectif et fait miroiter des projets à l'échelle internationale : 
Je vous ai fait jouer au Casino de Paris devant des salles pleines. Je vous considère comme une famille. Je vous ai pas invités à mon mariage? Mais c'est ça, on est une famille. Tout est lié. J'ai plein de projets pour vous. Ce matin j'avais encore Eddy Murphy au téléphone qui me disait qu'il a aimé le Jamel Comedy Club sur YouTube. Et vous me parlez d'argent? Alors ceux qui veulent continuer à faire des tournées, à jouer devant des salles pleines, je les excuse; les autres, vous pouvez partir.

La scène répète les techniques de culpabilisation à même de décourager l'expression d'un mécontentement ou d'un ressentiment. La sélection des propos, leur concentration, le déséquilibre entre le producteur tout puissant et la troupe silencieuse font basculer le récit vraisemblable du côté d'une représentation hyperbolique de la manipulation des affects.

Le flou sémantique et énonciatif de la série qui résulte de l'alliance d'une théâtralité assumée et d'une forme documentaire complexifie le contrat de la série avec ses spectateurs. Il construit certes un contrat de divertissement, en s'appuyant sur trois niveaux de plaisir. Le premier est le dévoilement de l'intime à l'œuvre dans d'autres genres médiatiques tels que la téléréalité et/ou l'information people (Dubied, 2009; Dakhlia, 2009). Le second repose sur ce que Umberto Eco appelle la compétence intertextuelle du spectateur qui, en mobilisant ses connaissances sur l'émission et ses participants, peut en apprécier à la fois la répétition (la familiarité du personnage) et l'innovation (le fait que les personnages ne correspondent pas à l'image qu'ils renvoient sur scène) (Eco, 1994, p.17-18). Le troisième englobe l'activité de «braconnage» (Certeau, 1990) et de réinterprétation du texte, sans doute favorisée par le brouillage des genres. Mais le flou du dispositif propose également aux spectateurs une «connivence critique» (Charaudeau, 2007, p. 36) dont il est nécessaire de clarifier l'objet. Inside Jamel Comedy Club s'apparente à un pastiche de documentaire compris, selon la définition de Richard Dyer, au sens d'imitation d'un genre, qui se désigne par certains aspects en tant qu'imitation (Dyer, 2007, p. 22-25). Mais ce pastiche n'est pas la simple imitation stylistique d'une forme audiovisuelle connue des spectateurs : ce qu'il évalue et détourne à des fins humoristiques n'est pas le reportage, le making of ou les émissions de téléréalité mais l'objet filmé, à savoir les relations entre les membres de la troupe. À ce titre, comme nous allons le voir, la série dévoile le faux-semblant des vertus de la diversité que l'émission originelle promeut et, à travers lui, la persévérance des procédés de stéréotypisation.

\section{De l’identité aux stéréotypisations}

La série Inside Jamel Comedy Club cite, déplace et en même temps dévalue le régime de véridiction de l'émission de plateau Le Jamel Comedy Club. Celle-ci est le lieu d'une politique de représentation au sens fort d'affirmation 
identitaire et d'expression des différences dans un contexte républicain français universaliste. Pour cela, elle s'appuie sur le récit à la première personne d'expériences présentées comme vécues, et insiste à l'échelle collective sur la diversité des publics et des intervenants. La part revendicative de l'émission est cependant conditionnée par l'usage récurrent de la «vanne » visant les membres du public et les comédiens les plus marqués par leur appartenance ethnoraciale ou subculturelle (Quemener, 2010, p.14-16). Cette dernière constitue un gage de non-sérieux. De son côté, Inside Jamel Comedy Club fabrique à son tour une représentation donnée pour réelle des comportements et des personnages. Ce faisant, il concurrence l'authenticité revendiquée de l'émission de plateau et met en lumière sa fabrication à des fins scéniques et politiques. Dédo, par exemple, explique sans sourciller avoir choisi le personnage du fan de hard rock par dépit, après que celui de séducteur a été emprunté par un autre humoriste à succès, Frank Dubosc. Il brille dans l'épisode 4 par sa méconnaissance des groupes mythiques et une attitude nonchalante, bière à la main et tatouages aux bras, qui montre sa conception stéréotypée du rockeur. Cette mise en abîme du personnage scénique imprègne l'ensemble du scénario - Frédéric Chau se voit contraint, pour faire rire, d'adopter un accent chinois caricatural, Claudia est incitée à créer un univers exotique. Elle pointe l'échec du Jamel Comedy Club à résister aux attentes des publics et à prendre le contre-pied systématique des stéréotypes. Surtout, elle montre l'artificialité de la mise en scène de la diversité et de l'apparent consensus autour de la dénonciation des logiques d'exclusion.

La série substitue à la représentation de la diversité l'image d'un collectif constitué de personnes dénuées d'autodérision et d'empathie, miné par des querelles d'ego et des préjugés. Ici, l'identité et l'expérience subalternes s'effacent au profit d'un égoïsme défavorable à toute conscience politique, qui s'active dans les interactions entre les personnages. Pour en rendre compte, la série insiste sur les conséquences sociales de l'énonciation d'une identité, notamment avec la mise en scène de «stigmates invisibles» (Goffman, 1977). Blanche fait part de son identité juive à la suite d'une remarque antisémite de Fabrice Éboué, tandis que Dédo avoue son parcours d'ex-nazi lorsque son tatouage de croix gammée est découvert par les membres de la troupe. Ces deux exemples, qui font intervenir des personnages blancs, fonctionnent sur le mode de l'ironie. Blanche, la moins «marquée » et la moins exposée a priori aux exclusions, fait l'expérience de la violence immédiate qui s'exerce sur elle une fois son identité juive connue - elle se voit d'emblée renvoyée au stéréotype du juif avide d'argent. Honteux et devenu infréquentable pour ses camarades, Dédo tente en vain de faire retirer son tatouage dans le dernier épisode. Son cas tourne vite au ridicule et au pathétique : étiqueté à vie, il gère maladroitement au quotidien les réactions négatives que son signe d'appartenance suscite. En s'attachant aux relations entre les personnages, la série s'extrait 
par conséquent de la représentation additionnelle des identifications et des constructions de soi qui était le propre du Jamel Comedy Club, au profit de la monstration des manières dont la rencontre d'individualités multiples favorise l'expression des identités et augmente les risques d'un recroquevillement sur soi. Elle construit l'image d'une diversité, lieu d'exacerbation des différences individuelles, nourrie par et nourrissant les logiques d'exclusion.

L'instabilité de personnages successivement victimes et bourreaux, objets de stigmatisation et producteurs de stigmates devient le déclencheur de sentiments contradictoires des uns vis-à-vis des autres au sein la troupe. Reposant sur des effets de renversement et de grossissement permanents, elle expose les relations du quotidien au dévoilement d'une palette de procédés discursifs aboutissant à la production de stéréotypes, entendus au sens de processus de réduction d'une catégorie à certains traits s'inscrivant dans des hiérarchies symboliques et des rapports de pouvoir (Macé, 2007, p.72). Parmi ces procédés, le premier est sans doute le plus insidieux, puisqu'il consiste en l'affichage d'une relation objective au monde. Principalement associé aux personnages blancs de la série, il se caractérise par l'absence d'interrogation du point de vue situé. JF, dès son apparition, adopte la rhétorique justificative classique de l'homme blanc qui détourne l'attention de ses privilèges en revendiquant une origine sociale subalterne par la classe et le lieu d'habitation : "Moi aussi, je viens de la banlieue, et j'ai grandi avec des gens comme vous. » L'expression «des gens comme vous» manifeste une condescendance et une hiérarchie implicite entre «Blancs» et «non-Blancs». Elle conduit à un racisme basé sur l'exacerbation des différences culturelles et sociales. Après la mort de Frédéric Chau, JF s'en prend à Claudia qui lui reproche son apparente insensibilité : «Je mange des côtes de porc, je bois du vin moi. Je suis pas Africain moi! ]'ai pas besoin de montrer à tout le village que je souffre. »JF réduit autrui à des attitudes et des traits culturels manifestes et indépassables. Sous son regard, les minorités se trouvent «marquées» de manière excessive par leur ethnicité, tandis que les Blancs se voient associés à des attitudes jugées plus neutres et «non remarquables» (Brekhus, 2001, p. 247). La scène rend compte du processus par lequel la blanchité maintient son hégémonie en se construisant dans l'illusion de son détachement du monde social (Dyer, 1997, p.12; Cervulle, 2011). Le personnage de Blanche résume parfaitement la rhétorique caricaturale du Blanc: elle se présente d'emblée comme «sans origine» et se pose en exception par rapport à ses camarades.

Le second procédé, étroitement lié au premier, recouvre l'expression de ce que Stuart Hall identifie comme un racisme implicite à travers l'enchaînement d'éléments venant qualifier de façon réductrice un groupe social (Hall, 2008, p. 261). Il consiste en un processus de stéréotypisation classique, qui repose sur l'association d'un groupe ou d'une catégorie à un nombre restreint de caractéristiques. Il se manifeste dans les raisonnements grossiers de Fabrice Éboué. 
Les dialogues et l'enchaînement des propos mettent l'accent sur la logique interprétative à l'œuvre :

Fabrice Éboué [à propos de l'enchaînement des sketches] : Deux filles à la suite, ça le fait pas, ça fait tomber le spectacle...

Amelle : Tu es en train de dire que les filles sont moins drôles que les garçons.

Blanche: C'est ultra misogyne!

Fabrice Éboué : C'est pas moi qui le dit... C'est connu.

Amelle : Attends, Muriel Robin, Florence Foresti !

Éboué : C'est pas pareil, elles sont gouines!

Blanche: Foresti est pas gouine.

Wahid: Si, elle a les cheveux courts.

Fabrice Éboué : Merci Wahid.

Chaque réplique révèle l'articulation des éléments et active les chaînes de signification aboutissant à des représentations réductrices : cheveux courts et lesbianisme, femme et absence d'humour ou encore Afrique et exotisme, comme nous l'avons vu avec Claudia. À cette révélation de la formation des stéréotypes, ici par l'enchaînement des répliques, s'ajoute la monstration de leurs conséquences affectives dans la gestion quotidienne du groupe. Promu chef de troupe après le départ de Jamel Debbouze, Fabrice Éboué décide de retirer le sketch de Frédéric Chau, le seul «Chinois » du spectacle, sous le prétexte qu'il suscite moins de rires que les autres. Les protestations vaines des membres du groupe que cette décision provoque mettent alors à jour l'injustice subie et l'arbitraire de la justification.

Le troisième procédé renvoie aux fantasmes qui gouvernent l'appréhension des groupes sociaux. L'arrivée dans la troupe d'une personne de petite taille, Christophe, dans l'épisode 4, suscite tantôt une réaction de rejet, tantôt un fétichisme risible. Alors qu'Amelle s'était montrée rebutée par des fans en fauteuil roulant venus l'acclamer à la fin d'un spectacle, elle manifeste à l'égard de Christophe un enthousiasme inhabituel :

Attends, c'est trop mignon un nain... C'est comme le chien de Paris Hilton, il grandit pas, c'est pratique. Le seul truc qui est un peu relou, c'est qu'il s'appelle Christophe. C'est pas top pour un nain. Si j'avais un nain à moi, je l'appellerais un truc mignon. Je l'appellerais Wizi... C'est mignon Wizi.

Dans la suite de l'épisode, Amelle considère Christophe comme une sorte de jouet et va jusqu'à lui acheter des chaussures sans que celui-ci ait demandé quoi que ce soit. Le surnom de Wizi et l'attitude infantilisante qu'elle adopte sont une mise en forme des projections qui construisent les imaginaires sociaux : Christophe devient l'objet d'un désir et d'une fascination qui ignorent la complexité de son être et de son groupe (Hall, 1997, p. 263). L'ironie de la saynète repose sur l'aveuglement exagéré du personnage aux lubies gouvernées par des représentations simplificatrices. 
La diversité qui était le fer de lance du Jamel Comedy Club est ici abordée de manière ironique et dépeinte comme le berceau d'un individualisme destructeur des solidarités et conduisant à la possible instrumentalisation des différences. Aucune lutte et aucun événement ne sont à même de souder le groupe. En insistant sur la fragmentation interne du tissu collectif, le spectacle risible de ces conflits de personnes suggère que la rencontre de personnages aux identifications diverses ne s'accompagne pas nécessairement d'une réflexivité individuelle et collective à l'égard des stéréotypes et encore moins de leur disparition. Au contraire, elle conduit chacun à se recroqueviller sur son expérience ou son sentiment d'exclusion, voire à confronter cette expérience à celle des autres, laissant poindre les signes d'une entrée en concurrence des doléances des groupes sociaux. On peut s'interroger sur la réception de cette monstration des logiques d'exclusion par le biais d'une représentation ironique de la diversité, formulée par ceux-là mêmes qui s'en sont fait les promoteurs. En effet, la série adopte une «stratégie énonciative risquée» (Jullier, 2010, p. 293), en s'appuyant sur un récit au « troisième degré » pouvant brouiller la visée critique.

\section{Absence de rires et rires cathartiques}

Afin d'examiner cette question, nous proposons une étude exploratoire des commentaires des internautes sur le site de partage de vidéos YouTube. La série mise en ligne plusieurs mois après sa première diffusion - les épisodes 1 à 6 sont postés entre décembre 2009 et février 2010, les épisodes 7 et 8 sont inaccessibles - est disponible par tranches de 10 à 15 minutes, soit deux à trois parties par épisode, du fait des contraintes techniques du site. Pour chaque épisode, nous avons recensé en mai 2011 un total de 1610 commentaires émis par presque autant d'internautes, les épisodes 1 à 4 étant sans conteste les plus commentés, avec 300 à 400 commentaires par épisode. L'éclatement des commentaires suscités par la série et la fragmentation de la réception sur YouTube (Van Dijk, 2007; Granjon, Le Foulgoc, 2010) empêchent l'identification d'un débat très suivi ou d'un public de fans (Jenkins, 1992, p.1-7). Ces commentaires ont néanmoins l'avantage d'exprimer par des émoticônes ou des abréviations type «ptdr» ou «mdr» des réactions telles que le rire ou le fou rire (Casilli, 2010, p.135), qui traduisent l'expérience immédiate du spectateur et l'activité interprétative à l'œuvre (Jenkins, 2006, p.45-51; Esquenazi, 2011). En l'absence de forums substantiels sur la série, ils fournissent une matière à l'élaboration d'hypothèses sur la réception des enjeux de représentation de ce trouble énonciatif.

L'analyse de ces commentaires permet d'identifier deux types de réactions qui s'articulent autour de la croyance en la réalité des personnages et des scènes filmées. Une proportion faible mais significative de commentaires (environ une trentaine par épisode) se caractérise par une condamnation violente 
des relations de pouvoir et des attitudes stigmatisantes montrées à l'écran. Elle s'exprime par la formulation d'insultes à l'égard d'un personnage particulier, à l'instar d'Amelle : "Franchement, même si je suis Marocain, Amelle elle pue la merde sérieux. Il y a pas de mots pour la décrire [série d'insultes]. » (Neoo Viirus, avril 2011) 5 Elle s'accompagne parfois d'une explicitation des raisons de ce rejet. Citant le passage dans lequel Amelle refuse de faire la bise à des spectateurs en fauteuils roulants, Distro3D use de la métaphore de l'écœurement : "Le passage des handicapés franchement... Autant j'aime Jamel et le Comédie Club, autant c'est le genre de clip qui me degoûte... » (janvier 2011). Ces commentaires coïncident avec une appréhension de la série sur le mode du réel et de l'authenticité. Ils s'adressent indistinctement aux personnages et à l'humoriste et concernent à la fois les comédiens les moins connus du grand public et ceux dont l'image médiatique ne laisse pas supposer une telle attitude. À l'inverse de Fabrice Éboué et Thomas N'Gijol qui connaissent tous deux une carrière au cinéma, à la télévision et la radio, l'humoriste Amelle n'a depuis l'émission Le Jamel Comedy Club que peu de visibilité publique, ce qui semble favoriser l'expression d'une désapprobation. La découverte de la dimension fictive s'accompagne d'un soulagement, notamment lorsqu'elle touche une personnalité connue de la série. «Je croyais que c'était vrai, pour un instant j'ai eu peur [...] L'attitude de Jamel pourrait laisser croire que c'est une personne super égoïste.» (Justwoofus, janvier 2011)

Ces commentaires conduisent à envisager une réception sur un mode «documentarisant» (Odin, 2000, p. 57-58), signifiant la violence des rapports de domination par le biais du dégoût. Il faut cependant préciser que le visionnage fragmenté que constitue YouTube favorise le trouble entre documentaire et mocumentaire, « réalité » filmée ou scénarisation, en détournant le spectateur de la trame narrative globale (Wojcik, 2010, p.110). Il serait possible d'interroger la force performative de ces émotions et leurs « vertus » dans la formation des imaginaires et des actions (Esquenazi, 2004, p.56). La clarification de la dimension fictionnelle de la série offre une piste lorsqu'elle conduit à un déplacement de l'objet de la critique. Ainsi, après que les commentaires ont explicité la part fabriquée, plusieurs internautes transfèrent leur désapprobation de l'attitude d'Amelle vers l'objet du rire en s'interrogeant sur les limites de l'humour: "Perso, l’idée de rire sur des handicapés me gène un peu. » (Peetakaamer, août 2010); "Même si c'est de la comédie, le coup des handicapés, j’ai trouvé ça limite» (mrskeud, mars 2010). Les réactions vives, une fois resignifiées par les discussions en ligne, donnent lieu à un métadiscours sur l'humour exprimant de façon explicite un jugement à l'égard de son pouvoir performatif: «[...] Même si c'est de l’humour, Christophe (le personage du nain) nous

5. La date d'émission du commentaire a été recomposée à partir de la date de consultation (10 juin 2011) et des indications disponibles surYouTube : "posté il y un an et cinq semaines». 
fait comprendre une chose, c'est qu'il faut rire de soi même, assumer la différence» (Naagard, juin 2010). Une part de la réception consisterait à débattre des contours de l'humour et par là, des autoreprésentations et stéréotypisations auquel il participe.

Les commentaires les plus nombreux consistent en la répétition des dialogues et répliques clés de la série. Ils expriment à la fois le plaisir du texte, de l'exagération humoristique et du rire qu'ils suscitent et signalent les dialogues les plus réducteurs. Ce type de réception coïncide avec l'appréhension du programme en tant que fiction. À l'inverse de la posture de rejet exposée plus haut, il touche principalement les comédiens dont l'image médiatique est imprégnée d'une forme d'exagération, tel Fabrice Éboué, commentateur controversé de l'émission de talk-show de M6, T'empêches tout le monde de dormir, entre 2006 et 2008. Les commentaires confondent dans ce cas plaisir du texte et évaluation du jeu d'acteur. Ainsi, à l'instar de KyleXyo, de nombreux internautes insistent sur le fait que les comédiens « jouent super bien », tandis que d'autres voient dans le pastiche de documentaire la preuve de leur «talent». Ce niveau de plaisir s'articule à celui de l'efficacité des répliques et des rôles attribués à chacun : "Je sais pas pour vous, mais moi Fabrice Éboué me TUE à chaque phrase qu'il prononce!!!!! ( (llcoolk456, octobre 2010); " Fabrice Éboué, j’ai envie de la claquer, mdr» (Aimessdu67, juin 2010). Ces commentaires expriment par une série d'abréviations et d'émoticônes un rire conclusif par lequel les internautes reconnaissent l'aspect fabriqué et surtout caricatural de la série.

Ce deuxième type de commentaires conduit à formuler l'hypothèse d'une réception sur le mode "spectacularisant», qui amène les spectateurs à évaluer ce qui est donné à voir (Odin, 2000, p. 66-67). Celle-ci consiste en une répétition distanciée des stéréotypes dont la part d'artifice et de théâtralité est sue. Cette distance se traduit dans les commentaires par des paroles empathiques formulées à l'égard des personnes destinataires des répliques les plus discriminantes dont elles signifient la violence : «HAHAHHA Fabrice quel tyran!! "Tu te fous de ma gueule ou quoi? Depuis hier je te dis qu'on travaille le thème de l'Africaine, et tu te mets à faire des régimes, à maigrir!" Pauvre Claudia! » (maggie27311, septembre 2010). Lorsqu'elle ne s'accompagne pas d'une marque d'empathie, la retranscription simple traduit une réaction partagée entre la distanciation et le plaisir de la réplique tabou car raciste, à l'instar de celle de Fabrice Éboué : «ll avait quel âge ton grand père? 77 ans. Pour l'Afrique c'est déjà pas mal.» (orman128, décembre 2009), ou de celle de Wahid à l'annonce de la judaïté de Blanche : « “C'est marrant, quand j't'ai vue, j'ai cru que t'étais en galère de tunes", PTDRRRRRRRRRR.» (K3D91, juillet 2010) Outre une sensation de divertissement, cette répétition est le moyen par lequel les internautes signalent à la fois les moments d'exagération et les logiques interprétatives réductrices mobilisées par les personnages, dont ils révèlent l'arbitraire 
(Butler, 1997, p.101). Elle dénote également une dimension cathartique, qui se constitue du plaisir de la goujaterie, de l'excès, de la réplique acerbe et de l'appropriation consciente des répliques les plus stéréotypées.

Dans les commentaires d'actualité, au cinéma comme au café-théâtre, le trouble énonciatif est un des procédés privilégiés par l'humour contemporain pour ouvrir un espace de provocation et de critique à l'égard des discours institutionnels et politiques. Cette forme humoristique participe certes d'une «contredémocratie » par une activité de surveillance, d'évaluation et de dénonciation (Rosanvallon, 2006, p.33). Mais sa visée potentiellement critique, voire politique, se heurte au flou du message et de l'intention des auteurs, ainsi qu'à une multiplicité de répliques et de renversements nécessaires à son maintien, qui souvent morcelle le propos au profit d'un rire systématique. Avec la série Inside Jamel Comedy Club, les deux modalités de réception, sentiments de dégoût ou citation exutoire des rhétoriques discriminatoires, traduisent des réactions émotionnelles et ponctuelles, favorisées par le dispositif numérique de commentaires en ligne. Elles ne s'accompagnent pas moins du signalement de la violence des stéréotypes et de leurs procédés discursifs. Elles invitent à l'exploration des échos éventuels aux discriminations passées et des contrecoups dans l'engagement des publics vers l'élaboration d'enjeux collectifs, notamment par le biais d'entretiens avec des spectateurs.

\section{Références}

BAKHTIne Mikhaïl, 1970 [1965], L'œuvre de François Rabelais et la culture populaire au Moyen Âge et sous la Renaissance, A. Robel trad., Paris, Gallimard.

BrekHUS Wayne, 2005, "Une sociologie de l'invisibilité. Réorienter notre regard», Réseaux, $\mathrm{n}^{0} 129-130$, p. 243-272.

ButLer Judith, 1997, Excitable Speech. A Politics of the Performative, New York, Londres, Routledge.

CAsilli Antonio A., Coville Marion (entretien par), 2011, "Corps en ligne, corps hors ligne », Poli - Politique de l'image, nº 4, p. 127-146.

Certeau Michel (de), 1990, L'invention du quotidien, I, Arts de faire, Paris, Gallimard.

Cervulle Maxime, 2011, «L'Écran blanc. Diversité, rapports sociaux de « race » et sociologie des spectateurs de cinéma», thèse soutenue le 20 juin 2011 à l'Université Panthéon-Sorbonne Paris 1.

Charaudeau Patrick, 2006, "Des catégories pour l'humour?", Questions de Communication, nº 10, p. 19-43.

DAKHLIA Jamil, 2009, «Du populaire au populisme? Idéologie et négociation des valeurs dans la presse people française », Communication, vol.XXVII, $\mathrm{n}^{\circ} 1$, en ligne [http://communication.revues.org/index1268.html], consulté le 20 juin 2011.

Dubied Annick, 2009, "L’information-people. La célébrité racontée par la presse», 
Communication, vol.XXVII, nº1, en ligne [http://communication.revues.org/ index1351.html], consulté le 20 juin 2011.

DYER Richard, 1997, White, Londres, New York, Routledge.

- 2007, Pastiche, Londres, New York, Routledge.

Eco Umberto, 1994, «Innovation et répétition. Entre esthétique moderne et postmoderne », Réseaux, vol. XII, n68, p. 9-26.

ESquenAZI Jean-Pierre, 2004, "Vers la citoyenneté. L'étape de l'émotion», Mots. Les langages du politique, $\mathrm{n}^{\circ} 75$, en ligne [http://mots.revues.org/3183], consulté le 2 janvier 2011.

- 2011, "Quand un produit culturel industriel est-il une "œuvre politique"?», Réseaux, vol. III, nº167, p.189-208.

Foucault Michel, 1994, "Sexe, pouvoir et la politique de l'identité», Dits et écrits, 1954-1988, tome IV, Paris, Gallimard, p.735-746.

GofFMAN Erving, 1975 [1963], Stigmate. Les usages sociaux des handicaps, A. Kihm trad., Paris, Minuit (Le Sens commun).

Granjon Fabien, Le Foulgoc Aurélien, 2010, «Les usages sociaux de l'actualité. L'expérience médiatique des publics internautes», Réseaux, vol. XXVIII, nº160-161, p. 225-253.

GUNSTER Shane, 2005, «"All about nothing”. Difference, affect, and seinfeld », Television \& New Media, vol. VI, nº 2, p. 200-223.

HALL Stuart, 1997, "The spectacle of the "Other" ", Cultural Representations and Signifying Practices, S. Hall éd., Birmingham, The Open University, p. 223-291.

- 2008 [1995], «Dans le blanc des yeux. Idéologies racistes et médias», Identités et cultures. Politiques des cultural studies, M. Cervulle éd., C. Jaquet trad., Paris, Éditions Amsterdam, p. 259-264.

Hatchuel Sarah, 2009, "Le film de tournage shakespearien. Fiction documentarisée ou documentaire fictionnalisé? », Sillages critiques, n¹0, en ligne [http://sillagescritiques.revues.org/2015], consulté le 10 janvier 2012.

Jenkins Henry, 1992, Textual Poachers. Television Fans \& Participatory Culture, New York, Routledge.

- 2006, Convergence Culture. Where Old and New Media Collide, New York, New York University Press.

Jost François, 1995, "Le feint du monde », Réseaux, vol. XIII, nº 72, p.163-175.

- 2007, Le culte du banal. De Duchamp à la téléréalité, Paris, CNRS.

JULLIER Laurent, 2010, "Politiquement (in)correct. OSS 117 dans les forums de discussion d'AlloCiné », Studies in French Cinema, vol. X, nº 3, p. 289-301.

MACÉ Éric, 2007, "Des “minorités visibles” aux néo-stéréotypes. Les enjeux des régimes de monstration télévisuelle des différences ethnoraciales», Journal des anthropologues, hors-série : «Identités nationales d’État», p. 69-87.

ODIn Roger, 2000, "La question du public. Approche sémio-pragmatique », Réseaux, vol.XVIII, nº 99, p. 49-72.

Quemener Nelly, 2010, “"Mère black, père noir, je suis métis!” Conflits, résistances, visibilités des humoristes non blancs sur la scène télévisuelle en France», La (re) construction de l'identité par le pouvoir et les médias, Clermont-Ferrand, Presses 
universitaires Blaise Pascal, en ligne [http://www.msh-clermont.fr/article2294. html], consulté le 2 mars 2012.

Rosanvallon Pierre, 2006, La contre-démocratie, Paris, Le Seuil.

SCHULMAN Norma, 1994, "The house that Black built. Television stand-up comedy as minor discourse», Jounal of Popular Film and Television, juillet, vol.XXII, nº 3 , p. 108-115.

Sepulchre Sarah, 2007, «Ego-fiction. La riposte de la fiction face à la téléréalité? Le mélange de fiction et de réalité dans Curb Your Enthusiasm et Fat Actress », Communication, vol.XXVI, n ${ }^{\circ} 1$, en ligne [http://communication.revues.org/index877. html], consulté le 20 juin 2011.

VAN DIJCK José, «Television 2.o. YouTube and the emergence of homecasting », Creativity, Ownership and Collaboration in the Digital Age, en ligne [http://web.mit.edu/commforum/mit5/papers/vanDijck_Television2.o.article], consulté le 2 janvier 2012.

WARIGODA Miriam, 2010, Le making-of artistique. Ses caractéristiques particulières et son rapport avec l'idéaltype, mémoire de maîtrise soutenu en février 2010 à l'Université du Québec à Montréal.

Woנcık Stéphanie, 2010, "La démocratie électronique, mythe et réalité», La démocratie. Histoire, théories, pratiques, J.-V. Holeindre, B. Richard éd., Paris, Sciences humaines, p.107-113. 